\title{
Akt-the Mammalian Target of Rapamycin (mTOR) Pathway Inhibition Increases Cervical Cancer Cell Chemosensitivity to Active Form of Irinotecan (SN-38)
}

\author{
Leri Septiani, ${ }^{1}$ Yudi Mulyana Hidayat, ${ }^{1}$ Yusuf Sulaeman Effendi, ${ }^{1}$ Tono Djuwantono, ${ }^{1}$ Dimas Erlangga \\ Luftimas, $^{2}$ Ahmad Faried ${ }^{2}$ \\ ${ }^{1}$ Department of Obstetrics and Gynecology, Faculty of Medicine, Universitas Padjadjaran-Dr. Hasan Sadikin \\ General Hospital \\ ${ }^{2}$ Oncology and Stem Cell Working Group, Health Research Unit, Faculty of Medicine, Universitas Padjadjaran- \\ Dr. Hasan Sadikin General Hospital
}

\begin{tabular}{|c|c|}
\hline \multirow[t]{3}{*}{ Abstract } & $\begin{array}{l}\text { Objective: To investigate the molecular pathway of the cytotoxic effect of SN-38 } \\
\text { in human cervical cancer cell lines. }\end{array}$ \\
\hline & $\begin{array}{l}\text { Methods: Two human cervical cancer cell lines were treated with various } \\
\text { concentrations of irinotecan for } 24-72 \text { hours and the sensitivity was analysed } \\
\text { using the MTT assay. Apoptosis was further observed through microscopic } \\
\text { examinations. The protein expression was determined using Western blot } \\
\text { analysis. }\end{array}$ \\
\hline & $\begin{array}{l}\text { Results: CaSki cells demonstrated the highest sensitivity to SN-38, whereas } \\
\text { HeLa cells showed the lowest. In cervical cancer cells, SN-38 induced apoptosis } \\
\text { through an intrinsic- and extrinsic-pathways. In addition, we showed that SN-38 } \\
\text { downregulated the phosphorylation of Akt-mTOR pathways in CaSki cells, but not } \\
\text { in HeLa cells. Interestingly, in HeLa cells, which were more suggestive of a resistant } \\
\text { phenotype, pre-treatment with LY294002 and rapamycin inhibited activation of } \\
\text { Akt-mTOR signaling and significantly enhanced the sensitivity of HeLa cells to SN- } \\
38 \text {. }\end{array}$ \\
\hline $\begin{array}{l}\text { Received: } \\
\text { October 31, } 2012\end{array}$ & $\begin{array}{l}\text { Conclusions: Irinotecan exerts its anti-neoplastic effects on cervical cancer } \\
\text { cells by inducing apoptosis through caspase-cascade. Inhibition of Akt-mTOR, } \\
\text { LY294002 and rapamycin, which is targeted to Akt-mTOR pathways, may sensitize } \\
\text { irinotecan-resistant cervical cancer cells. }\end{array}$ \\
\hline $\begin{array}{l}\text { Revised: } \\
\text { February 1, } 2013\end{array}$ & $\begin{array}{l}\text { Keywords: Akt-mTOR pathways anti-neoplastic drugs, cervix cancer cells, } \\
\text { LY294002, rapamycin }\end{array}$ \\
\hline $\begin{array}{l}\text { Accepted: } \\
\text { February 24, } 2013\end{array}$ & IJIHS. 2013;1(1):13-21 \\
\hline
\end{tabular}

\section{Introduction}

Carcinoma of the cervix is the second most common neoplasm in Indonesian women and the second most common neoplasm leading to female cancer mortality. ${ }^{1}$ Furthermore, it is also the second most frequently found cancer among women in reproductive age between 1544 years old. ${ }^{2}$ It constitutes almost 15,000 new cases and 7,500 deaths per annum. ${ }^{2}$

Cervical cancer treatment depends on the Fédération Internationale de Gynécologie et d'Obstétrique (FIGO) staging. Early stadium of

\footnotetext{
Correspondence:

Leri Septiani, Department of Obstetrics and Gynecology, Faculty of Medicine, Universitas Padjadjaran-Dr. Hasan Sadikin General Hospital

Jl. Pasteur No. 38, Bandung, Indonesia e-mail: Isfaried@gmail.com
}

cervical cancer is generally treated with radical operations but up to now there is no fixed standard therapy for FIGO stadium Ib-lla of cervical cancer. Cancer within these stadiums can be treated with radical operation, radiotherapy, surgery-radiotherapy combination, surgeryanticancer drug combination, or chemotherapy although these combination therapy protocols may vary. The administration of the combination therapy for each patient is based on many factors including age, general condition, and patient's preference to achieve maximum therapy target with minimum complications.

The standard surgery for cervical cancer in stadium Ib and Ila is radical hysterectomy with pelvic lymphadenectomy. The benefit of surgical therapy is the ability to extract primary tumor and apply staging accurately which leads to the 
selection of the most appropriate type of adjuvant therapy for the patient. For stadium Ib2 and Ila, neoadjuvant chemotherapy is one of several adjuvant therapy alternatives applied. The goal of this neoadjuvant chemotherapy is to eradicate micrometastasis and reduce primary tumor volume so the next treatment effectiveness can be improved both for surgery and radiotherapy. In addition to radiotherapy, chemotherapy is the standard treatment for advanced cervical cancers. However, the systemic therapy protocol for this kind of cervical cancer keeps changing. In the past, chemotherapy was used for recurrent and persistent cervical cancer, or cervical cancers with metastasis. Currently, chemotherapy is the primary treatment for cervical cancers with a high risk of reccurrency.

The best possible treatment of locally advanced cervical cancer is a combination of radiation and cisplatin-based chemotherapy. Cisplatin-based chemotherapy as the main regimen is still the primary choice for advanced, metastatic, and recurrent cervical cancers. Even with this regimen, the response rate of advanced stadium patients was only $23 \%$ with limited response time. ${ }^{3}$ From this data, it is suggested that conventional treatment methods have reached a plateau and, therefore, to overcome this problem, finding a good prognostic factor and predictor response to chemotherapy might be useful.

Irinotecan (CPT-11) and its analog have given promising results on many clinical trials. SN-38, which is a semi-synthetic derivative of camptothecin, is the active metabolite of Irinotecan. Like other camptothecin-derivatives, SN-38 inhibits topoisomerase I (Topo I), a nuclear enzyme needed for replication and transcription by unwinding supercoiled DNA. ${ }^{4}$ SN-38 interferes with Topo I activity by trapping Topo I-DNA cleavage complexes, leading to lethal replication-mediated double strand breaks and ended with cell death. ${ }^{4}$ Currently, irinotecan is used as a standard therapy for colon cancer but not for cervical cancer. Irinotecan specificity against cervical cancer needs to be studied.

Chemotherapeutic agents kill cancer cells through interaction with several distinct intracellular targets, including factors affecting cells cycles, apoptosis cell death, and survival pathways. Class I phosphatidylinositol 3-kinase (PI3K) and its downstream effectors, such as Akt and mTOR, have emerged as a prominent proliferation and survival pathway. Activation of the PI3K/Akt-mTOR pathway has been implicated in many human cancers and shown to be associated with chemoresistance. ${ }^{5}$ We have previously shown that advanced cervical cancer expressed Akt and mTOR and the expression of p-mTOR could predict the response to neoadjuvants and the survival of the patients treated with cisplatin-based neoadjuvant chemotherapy. ${ }^{6,7}$ A therapeutic strategy for selectively modulating the expression of a gene of interest is the use of inhibitor agents of Akt such as LY294002 and inhibitor agents of mTOR such as rapamycin. We assessed whether the active form of irinotecan treatment enhanced the inhibition of proliferation signals, in this case the Akt-mTOR pathway, which led to increase of cell death.

\section{Methods}

\section{Cell lines and culture conditions}

Two human cervical cancer cell lines, the HeLa cell line which is an adenoma cell carcinoma with human papilloma virus or HPV-18 (+) and CaSki cell line which is a squamous cell carcinoma with HPV-16 (+), were purchased from the American Type Culture Collection (ATCC) (Manassas, VA). HeLa cells were maintained in Eagle's Minimum Essential Medium (EMEM) obtained from Sigma Chemical Co. (St. Louis, MO), supplemented with $2 \mathrm{mM}$ L-glutamine, $1.0 \mathrm{mM}$ sodium pyruvate, and $10 \%$ heat-inactivated fetal bovine serum (FBS) obtained from Gibco (BRL, Grand Island, NY). The CaSki cells were maintained in Rosewell Park Memorial Institute-1640 (RPMI-1640) medium (Gibco) supplemented with HEPES (Sigma) and $10 \%$ heat-inactivated FBS.

\section{Drugs and cell treatment}

Irinotecan was purchased from Wako Pure Chemicals (Osaka, Japan) and received as sterile lyophilized powder. A stock solution of $5 \mathrm{mg} /$ $\mathrm{mL}$ was made in dimethylsulfoxide (DMSO) and stored at $4{ }^{\circ} \mathrm{C}$. Further dilutions were made in culture media to reach the desired concentrations when the cells obtained approximately $80 \%$ confluency. The final concentration of DMSO did not exceed $0.08 \%$, a concentration that does not alter the growth or survival properties of any cell type. We used CPT-11 and SN-38 stock of $3 \mu \mathrm{M}$ peak plasma concentration (ppc) with a range of $9.8 \mathrm{ng} / \mathrm{mL}-5$ HM. LY294002 and rapamycin were purchased from Calbiochem (San Diego, CA). For LY294002 (25 $\mu \mathrm{M})$ or rapamycin (100 nm), cells were pre-incubated with these inhibitors for 6 hours (h) prior to irinotecan treatment.

\section{Drug sensitivity assay}

Cell proliferation analysis was performed on human cervical cancer cell lines in the presence of various concentrations of drugs by a colorimetric 
methyl thiazolyl tetrazolium (MTT) assay. Briefly, exponentially growing cells (2x104 cells/well) were plated in 96-well plates. After an overnight culture, the medium was substituted by a fresh medium with different concentrations of drugs. At the end of various treatments, $10 \mu \mathrm{L}$ of a cellcounting solution (WST-8, Dojindo Labs, Tokyo, Japan) was added. After dissolving the crystals with $1 \mathrm{~N} \mathrm{HCl}$-isopropanol, the absorbance was measured at a wavelength of $450 \mathrm{~nm}$ using a microtiter plate reader (Beckton Dickinson, Franklin Lakes, NJ). A value of $100 \%$ was assigned to untreated control and the concentration of drug that reduced the number of viable cells to $50 \%$ after $24 \mathrm{~h}$ of exposure $\left(\mathrm{CPI}_{50}\right)$ was derived from cell survival plots. All experiments were performed in triplicate.

\section{Light microscopy examination}

All cells were cultured in a $6-\mathrm{cm}$ plate and treated with drugs as described previously. Morphological changes were examined at the times indicated and photographed using a regular phase contrast microscope.

Western blot analysis

Western blot analysis was applied as described..$^{5}$ Cells were harvested at the time indicated and prepared in a buffer (20 mM Tris- $\mathrm{HCl}, \mathrm{pH} 7.6,1$
mM EDTA, 140 mM NaCl, 1\% Nonidet P-40, 1\% aprotinin, $1 \mathrm{mM}$ phenylmethylsulfonylfluoride, and $1 \mathrm{mM}$ sodium vanadate). The concentration of the sample protein was determined by bicinchoninic acid (BCA) protein assay (Pierce, Rockford, IL). Equal amounts of protein $(40 \mu \mathrm{g})$ were subjected to a 5-20\% Tris-tricine Ready Gel (Bio-Rad, Tokyo, Japan) and then transferred to nitrocellulose membrane (Amersham Pharmacia Biotech, Buckinghamshire, UK). Membrane was blocked with Tris-buffered saline/0.1\% Tween 20 with $5 \%$ nonfat dry milk and incubated in primary antibodies against caspase-3, caspase-8, caspase-9 and PARP (Santa Cruz); phosphorylated Akt (p-Akt, Ser473), phosphorylated mTOR (p-mTOR, ser2448) and phosphorylated p70 S6 Kinase (pS6K1,Thr389) from Cell Signaling at $4{ }^{\circ} \mathrm{C}$ overnight. The bands were visualised by a chemiluminescence system (Amersham). The expression of beta-actin (Sigma) served as a loading control. The density of the bands was quantified using Quantity One (BioRad).

\section{Statistical analysis}

Quantitative experiments were analyzed using Student's $t$ test. The P value was gained from the two-sided tests and were considered significant when $p<0.05$.
(A)

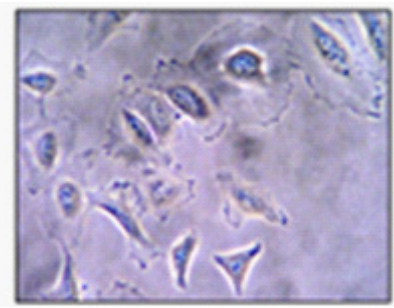

(B)

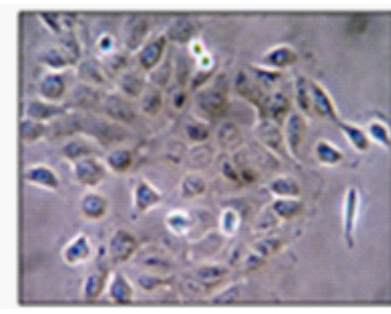

2 hours

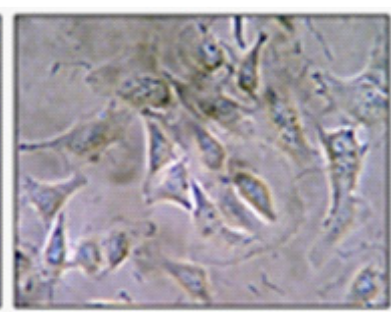

2 hours

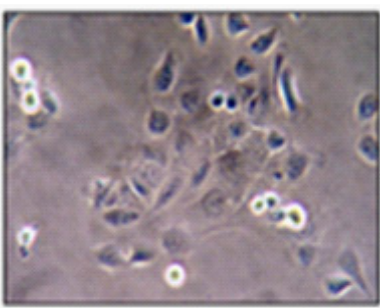

24 hours

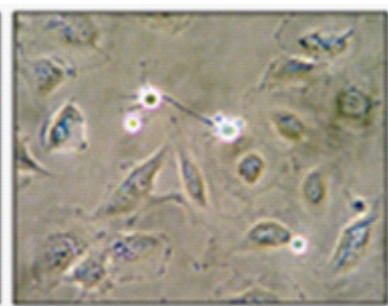

24 hours

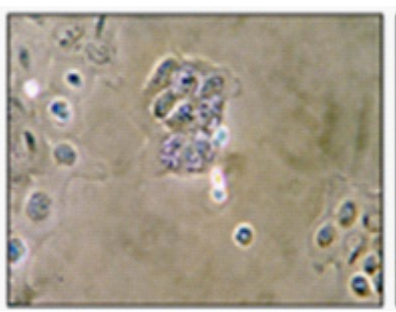

36 hours

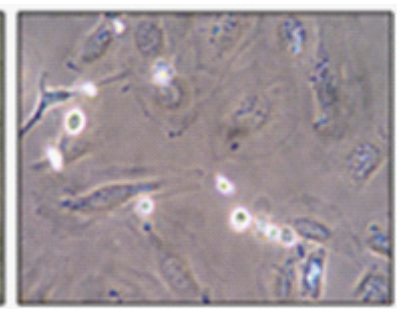

36 hours

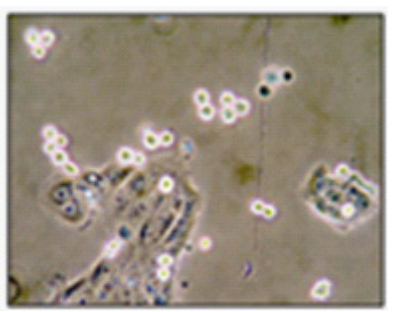

Fig. 1 Morphological Assessment of HeLa (A) and CaSki Cells (B) in Control Cultures and after SN-38 Treatment in Time-dependent Manner Using Phase-contrast Microscopy (200x Magnification) 

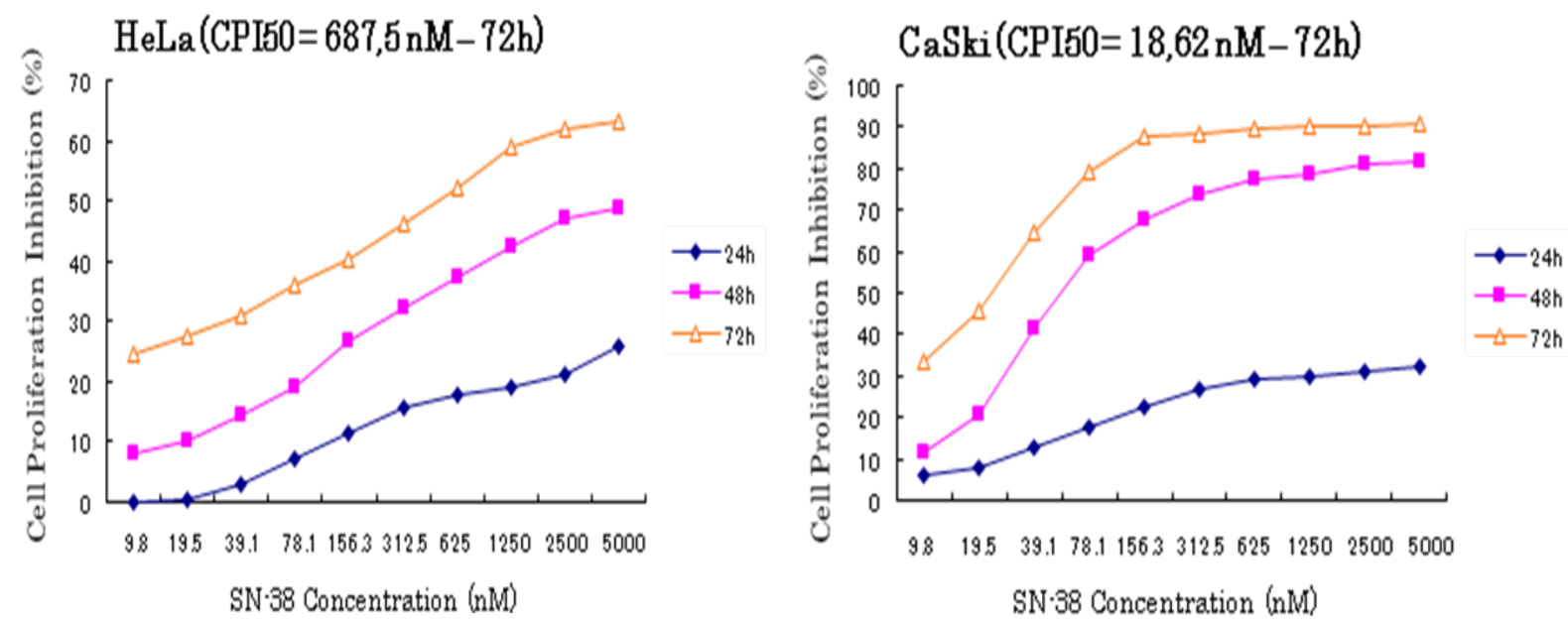

Fig. 2 The Effect of SN-38 on the Growth Inhibition of HeLa (Left) and CaSki (Right) Cells Using MTT Assay. Concentration-and Time-related Inhibition of Cells Growth at 24, 48 and 72 Hours were Shown

\section{Results}

Sensitivity of human cervical cancer cells to CPT11 and $\mathrm{SN}-38$

To investigate the sensitivity of human cervical cancer cells to CPT-11 and SN-38, the cells were treated for $24-72 \mathrm{~h}$ in a medium containing various concentrations of drugs. The exposure to drugs $(9,8 \mathrm{ng} / \mathrm{mL}-5 \mu \mathrm{M})$ produced a dose and time dependent cell growth reduction in HeLa and CaSki cells (Fig. 1-2). CaSki cells demonstrated a higher sensitivity compared to HeLa. The cell proliferation inhibition $50\left(\mathrm{CPI}_{50}\right)$ values for $72 \mathrm{~h}$ treated HeLa were $320 \mu \mathrm{M}, 1.91 \mu \mathrm{M}$ and 687.5
nM for CPT-11, SN-38 disolved with medium, and $\mathrm{SN}-38$ disolved with pure water $\left(\mathrm{H}_{2} \mathrm{O}\right)$, respectively. On the other hand, for $72 \mathrm{~h}$ treated CaSki, the results were $224 \mu \mathrm{M}, 50.8 \mathrm{nM}$ and 18.6 nM for CPT-11, SN-38 disolved with medium, and $\mathrm{SN}-38$ disolved with pure water, respectively (Table 1). Based on these results, both HeLa and CaSki cells treated in SN-38 disolved with pure water were used for further experiments.

Expression and modulation of other apoptosisrelated proteins by SN-38

In this study, it is also observed that SN-38 treatment had significantly upregulated the

Table 1 Concentration Proliferation Inhibition $50\left(\mathrm{CPI}_{50}\right)$ of HeLa and CaSki Cells to Rinotecan, SN-38 Disolved with Medium, and SN-38 with Pure Water $\left(\mathrm{H}_{2} \mathrm{O}\right)$

\begin{tabular}{cccc}
\hline Cell type & Irinotecan & SN-38 (med) & SN-38 (H2O) \\
\hline HeLa & $320 \mu \mathrm{M}(72 \mathrm{~h})$ & $1.91 \mu \mathrm{M}(72 \mathrm{~h})$ & $687.5 \mathrm{nM}(72 \mathrm{~h})$ \\
CaSki & $224 \mu \mathrm{M}(72 \mathrm{~h})$ & $50.8 \mathrm{nM}(72 \mathrm{~h})$ & $18.62 \mathrm{nM}(72 \mathrm{~h})$ \\
\hline
\end{tabular}

Table 2 Concentration Proliferation Inhibition $\left(\mathrm{CPI}_{50}\right)$ of HeLa and CaSki Cells to SN-38 Alone, with Pre-treatment LY294002 (LY), and Rapamycin (Rap)

\begin{tabular}{cccc}
\hline Cell type & SN-38 & LY-SN-38 & Rap-SN-38 \\
\hline HeLa & $687.5 \mathrm{nM}$ & $265.63 \mathrm{nM}$ & $181.6 \mathrm{nM}$ \\
CaSki & $18.62 \mathrm{nM}$ & $15.68 \mathrm{nM}$ & $4.9 \mathrm{nM}$ \\
\hline
\end{tabular}

LY, LY294002; Rap, rapamycin 
activation of caspase-8, caspase-9 (35 kDa and $37 \mathrm{kDa}$ ), and caspase-3 (19 kDa and $17 \mathrm{kDa}$ ) followed by the cleavage of its $89 \mathrm{kDa}$ substrate protein with PARP as a hallmark of apoptosis in CaSki cells (Fig. 3). This was seen as early as 2 hours and increased in a time dependent manner, demonstrating a significant role of $\mathrm{SN}-38$ induced apoptosis. On the other hand, the HeLa cells show that there are not much modification of active forms of caspase-cascade and PARP's expression (it reaches the plateu phase after 2 hours of treatment) found. These results indicate that SN-38 induced apoptosis differently in both cervical cancer cells. CaSki cells are more sensitive to SN-38 compared to HeLa cells.

SN-38 treatment altered the expression of PI3K/ Akt-signaling proteins

One of the most important survival-signaling pathways is mediated by PI3K and its downstream targets, such as Akt and mTOR. ${ }^{8}$ It was recently reported that Akt plays an important role in determining the chemosensitivity of many kinds of cells. ${ }^{9-11}$ We evaluated the expression of these survival signaling proteins in response to $\mathrm{SN}-38$ exposure in cervical cancer cells. It showed that SN-38 inhibited the activation of Akt in CaSki cells in dose and time dependent manner as shown by the reduced expression of active form or phosphorylated Akt (Ser473; 60 $\mathrm{kDa}$ ), phosphorylated mTOR (Ser2448; $289 \mathrm{kDa})$ and phosphorylated p70 S6 Kinase (Thr389; 70 kDa) (Fig. 4). On the other hand, for HeLa cells, there is not much modification of the active form Akt (p-Akt), mTOR (p-mTOR) and pS6K1 expression. These results indicate that SN38 downregulated differently in both cervical cancer cells. CaSki cells are more sensitive to SN-38 treatment compared to HeLa cells. These results indicate that the Akt-mTOR pathway

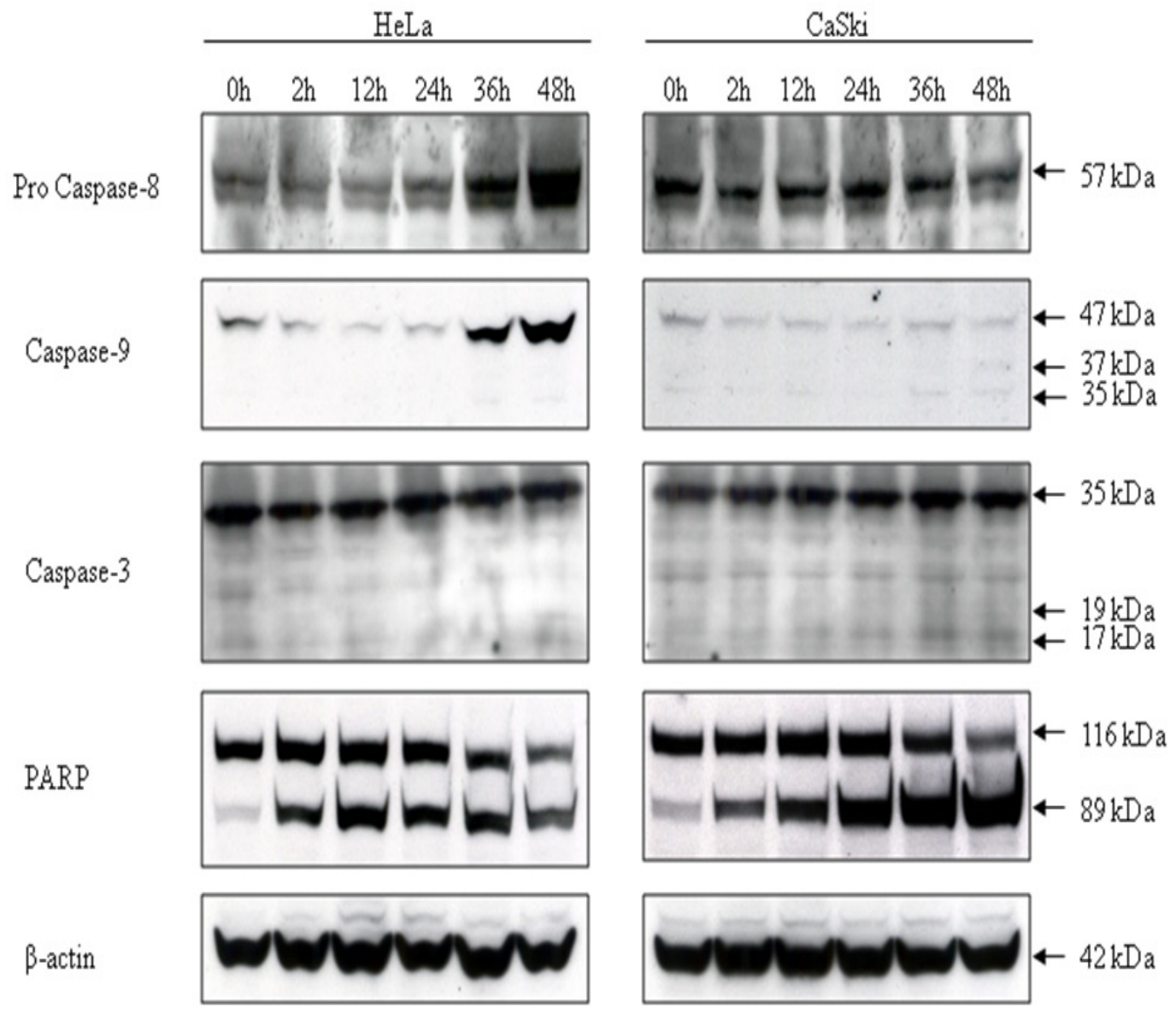

Fig. 3 SN-38 Induced Apoptosis; Western Blot Analysis of Active Form of Caspase-cascade and PARP Expression in HeLa and CaSki Cells Treated with SN-38. The Expression of $\beta$-actin Served as the Loading Control 
(A)

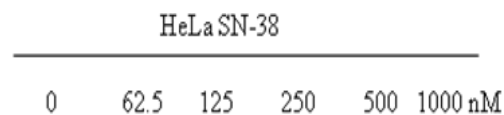

p-Akt

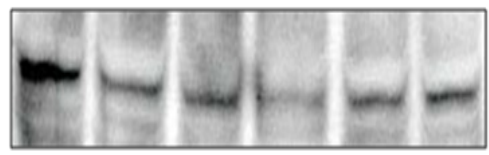

p-mTOR

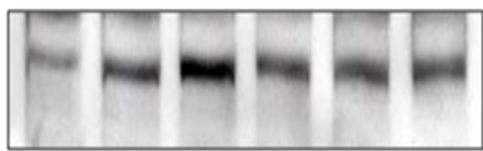

p 70 S6K

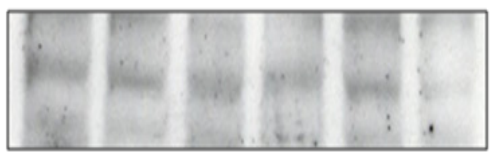

$\beta$-actin

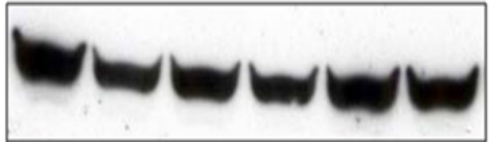

(B)

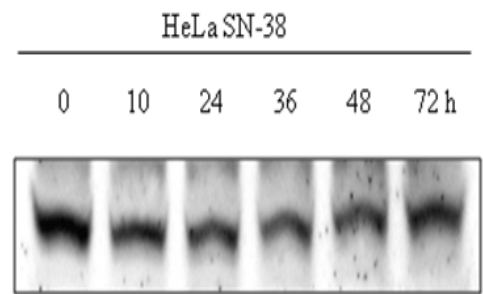

p-mTOR

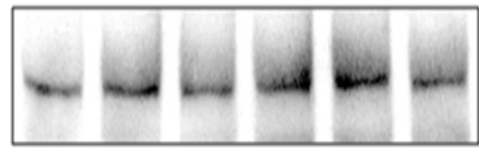

p70S6K

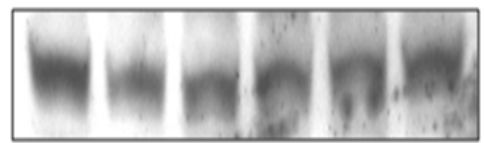

$\beta$-actin

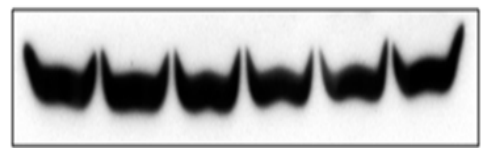

Caski SN-38
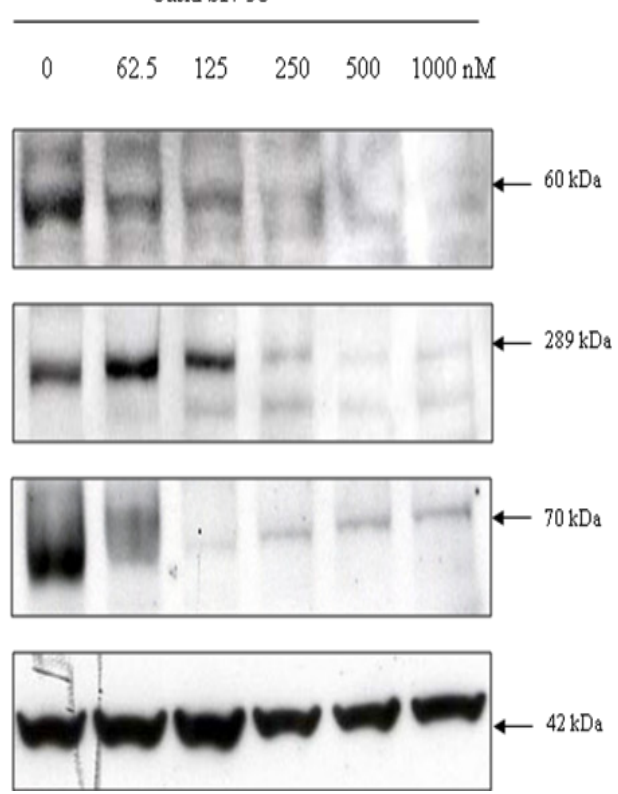

Caski SN-38
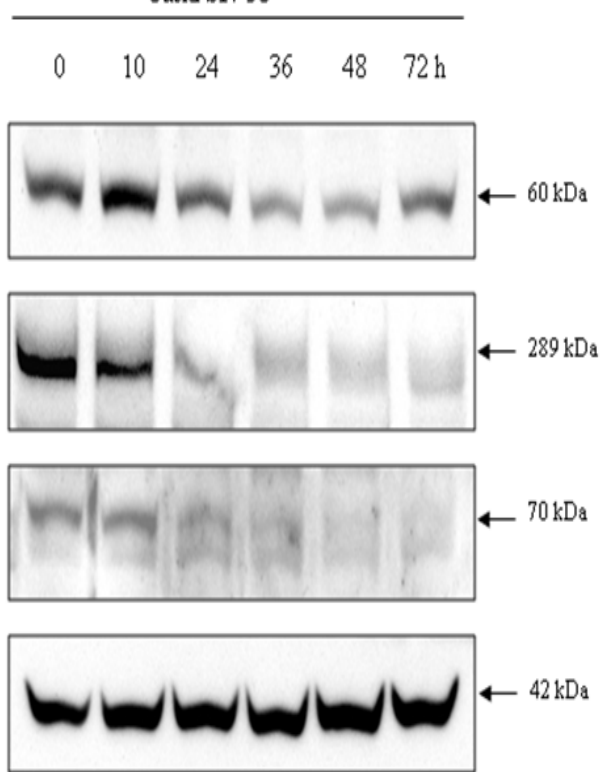

Fig. 4 SN-38 Effect on p-Akt, p-mTOR and p-70S6K1 Western Blot Analysis of Active Form Akt-mTOR Pathways in HeLa and CaSki Cells Harvested for the Time Indicated (A) and Different Dose (B). $\beta$-actin Served as Control

might have an important role to maintain the survival of CaSki cells in response to SN-38.

Inhibition of Akt-mTOR pathway increases the sensitivity of SN-38 induced apoptosis

To confirm whether the Akt-mTOR pathway plays a role in response to SN-38, the LY294002 and rapamycin as pretreatment to irinotecan were used. Pre-treatment with LY294002 and rapamycin significantly enhance the cell proliferation inhibition in HeLa cells $(p<0.05$, Table 2, Fig. 5). As in CaSki cells, pre-treatment with LY294002 did not significantly enhance the cell proliferation inhibition. On the other hand, 
pre-treatment with rapamycin significantly enhanced the chemosensitivity of CaSki cells to SN-38 ( $p<0.05$, Table 2). To further evaluate the effect of pre-treatment with these inhibitor agents on apoptosis, an analysis on the morphological changes were performed using phase contrast microscopy. The results show that pre-treatment with rapamycin induced apoptosis in most of CaSki cells, which appeared as bright, rounded, and detached cells from the dish.

\section{Discussion}

Irinotecan, which is one of the anti-neoplastic agents with the broadest spectrum, is currently used in the treatment of many types of advanced cancers, but not in the cervical carcinoma. Until now, the prognosis of patients with advanced, persistent, or recurrent squamous cell carcinoma of the cervix has been poor. ${ }^{1,2}$ Resistance to chemotherapy is the most frequent obstacle to effective treatment. Although the molecular mechanisms of paclitaxel in the mediation of cell death are well characterised, its effects on survival signaling remain unclear. This study determined the sensitivity of human cervical cancer cells to irinotecan (CРT-11) as well as its active form (SN-38) and the results from the MTT assay showed that CaSki cells had the highest sensitivity, whereas HeLa cells was the most resistant. Furthermore, an examination on whether CPT-11 and SN-38 induced apoptosis in human cervical cancer cells was also performed. To determine the molecular mechanism that mediates the cytotoxic effect of SN-38 in human cervical cancer cells, we performed Western blot analysis. In this study, it is demonstrated that SN38 activated caspase- 8 , caspase- 9 , caspase- 3 and PARP in CaSki cells. These results indicate that in sensitive CaSki cells the induction of apoptosis by SN-38 might take place through intrinsic and extrinsic pathways.

It was recently reported that CPT-11 and $\mathrm{SN}$ 38 activate a serine/threonin protein kinase Akt, which is the downstream target of $\mathrm{PI} 3 \mathrm{~K}^{10,12}$ Our results demonstrated that $\mathrm{SN}-38$ inhibits the activation of Akt in CaSki cells, which were more suggestive of a sensitive phenotype, which is shown by the downregulation of phosphorylated Akt at serine 473, phosphorylated mTOR at Ser2448, and phosphorylated p70 S6 Kinase at Thr389. Akt-mTOR pathways have the main role in promoting cell survival and are frequently associated with chemoresistance to cytotoxic drugs. The elevation level of phosphorylated Akt-mTOR pathways was reported to be involved in the mechanism of chemoresistance. ${ }^{13}$ Surprisingly, however, in the HeLa cells, which were more suggestive of a resistance phenotype,
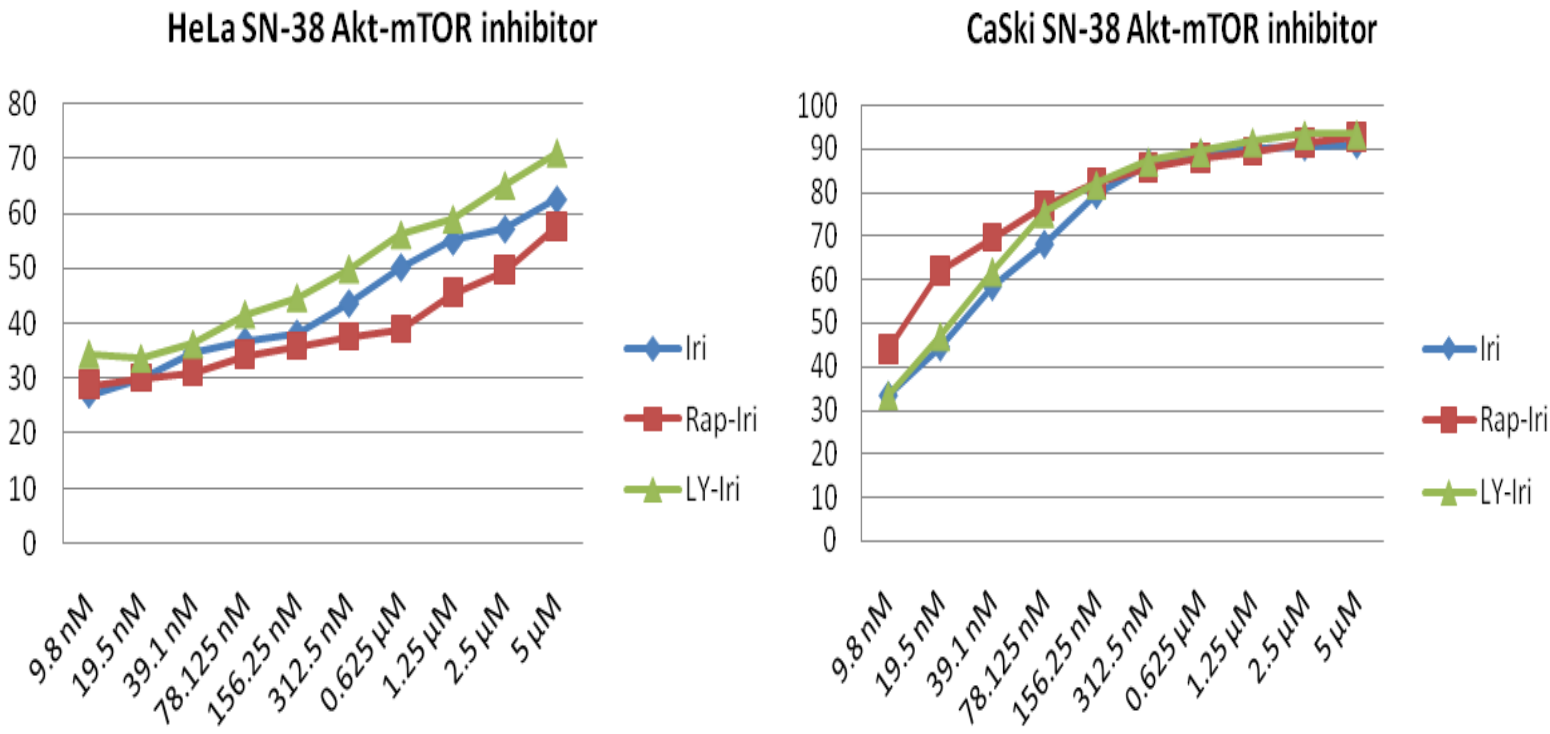

Fig. 5 Akt-Mtor Inhibitor Enhances Chemosensitivity of Hela (Left) and Caski (Right) Cells to Sn-38; Cells Treated with Sn-38 Alone, with or without $25 \mu \mathrm{M}$ Ly294002 or $100 \mu \mathrm{M}$ Rapamycin Pre-Treatment. The Cells Proliferation Inhibition was Determined by MTT Assay 
there was no altered activation of Akt-mTOR pathways to $\mathrm{SN}-38$ treatment. It is tempting to speculate that the Akt-mTOR inhibitor might have an important role in promoting the cell death of HeLa cells. To elucidate this phenomenon, we have conducted experiments using LY294002 or rapamycin as the Akt-mTOR inhibitor. We observed that the inhibition of Akt activation using LY294002 significantly enhance the sensitivity to $\mathrm{SN}-38$ in HeLa cells $(p<0.05)$. Furthermore, the inhibition of mTOR activation using rapamycin more significantly enhance the sensitivity to SN-38 in HeLa cells $(p<0.01)$.

Rapamycin has been found to inhibit serine/threonin kinase mTOR by binding to the immunophilin FK506-binding protein 12 (FKBP12). The inhibition of mTOR kinase leads to dephosphorylation of its two major downstream signaling components, p70 S6 kinase (S6K1) and $4 \mathrm{E}-\mathrm{BP} 1{ }^{14}$ Furthermore, we used rapamycin to inhibit Akt-mTOR activation in both HeLa and CaSki cells. As expected, we found that the inhibition of Akt-mTOR significantly enhanced the sensitivity of HeLa cells to irinotecan. Analysis of the morphology of cells under lightmicroscopy strengthened our findings that the inhibition of Akt-mTOR effectively increased irinotecan-induced apoptosis. Taken together, our results suggest that Akt-mTOR and its downstream effectors, S6K1, play a role in the survival of HeLa cells and that the inhibition of mTOR signaling may substantially potentiate apoptosis.

In summary, the study presented here is the first to directly address the potential of targeting Akt-mTOR protein in the enhancement of therapeutic efficacy of a Topoisomerase I agent, irinotecan, in human cervical cancer cell lines. This information may increase our understanding to apply molecular targeting therapies that may improve the management of cancer with chemotherapy.

\section{Acknowledgment}

This work was supported by Grants-in-Aid for Post Doctoral Research (PHK-PKPD SK No. 853/ UN6.C/Kep/HK/2011) from the Indonesian Directorate General of Higher Education (DIKTI)Faculty of Medicine, Universitas Padjadjaran, Bandung (FK Unpad) for Leri Septiani.

Author Disclosure Statement Authors have no conflict of interests.

\section{References}

1. Ferlay J, Bray F, Pisani P, Parkin D. GLOBOCAN 2002. Cancer incidence, mortality, and prevalence worldwide. IARC Cancer Base No.5, version 2.0. Lyon: IARC Press; 2004.

2. Castellague $X$, de Sanjose $S$, Aguado T, Louie KS, Bruni L, Munoz J, et al. HPV and cervical cancer in the world: 2007 report. Vaccine [serial on the internet]. 2007 Nov [cited 2012 April 5]: 2007;25(Supp 3):[about 230]. Available from: www.who.int/hpvcentre.

3. Thigpen $T$, Vance RB, Khansur T. The platinum compounds and paclitaxel in the management of carcinomas of the endometrium and uterine cervix. Semin Oncol. 1995;22(5 Suppl 12):67-75.

4. Fiorica JV. The role of topotecan in the treatment of advanced cervical cancer. Gynecol Oncol. 2003;90(3 Pt 2):S16-21.

5. Faried LS, Faried A, Kanuma T, Nakazato T, Tamura $\mathrm{T}$, Kuwano $\mathrm{H}$, et al. Inhibition of the mammalian target of rapamycin (mTOR) by rapamycin increases chemosensitivity of CaSki cells to paclitaxel. Eur J Cancer. 2006;42(7):934-47.

6. Faried LS, Faried A, Kanuma T, Sano T, Nakazato $\mathrm{T}$, Tamamura $\mathrm{T}$, et al. Predictive and prognostic role of activated mammalian target of rapamycin in cervical cancer treated with cisplatinbased neoadjuvant chemotherapy. Oncol Rep. 2006;16(1):57-63.

7. Faried LS, Faried A, Kanuma T, Sano T, Nakazato $\mathrm{T}$, Tamura $\mathrm{T}$, et al. Expression of $\mathrm{p}-\mathrm{mTOR}$ in adenocarcinoma of cervix: a potential biomarker and molecular target therapy. Molecular Carcinogenesis. 2008;47(6):446-57.

8. McCormick F. Cancer: survival pathways meet their end. Nature. 2004;428(6980):267-9.

9. Nguyen DM, Chen GA, Reddy R, Tsai W, Schrump WD, Cole G Jr, et al. Potentiation of paclitaxel cytotoxicity in lung and esophageal cancer cells by pharmacologic inhibition of the phosphoinositide 3-kinase/protein kinase B (Akt)-mediated signaling pathway. J Thorac Cardiovasc Surg. 2004;127(2):365-75.

10. Mabuchi S, Ohmichi M, Kimura A, Hisamoto $\mathrm{K}$, Hayakawa J, Nishio $\mathrm{Y}$, et al. Inhibition of phosphorylation of BAD and Raf-1 by Akt sensitizes human ovarian cancer cells to paclitaxel. J Biol Chem. 2002;277(36):33490-500.

11. Fahy BN, Schlieman MG, Virudachalam S, Bold 
RJ. Inhibition of AKT abrogates chemotherapyinduced NFkB survival mechanisms: implications for therapy in pancreatic cancer. J Am Coll Surg. 2004;198:591-9.

12. Lin HL, Lui WY, Liu TY, Chi CW. Reversal of taxol resistance in hepatoma by cyclosporin $A$ : involvement of the PI-3 kinase-AKT1 pathway. $\mathrm{Br}$ J Cancer. 2003;88(6):973-80.
13. Page $C$, Lin $H J$, Jin $Y$, Castle VP, Nunez G, Huang $M$, et al. Overexpression of Akt/AKT can modulate chemotherapy-induced apoptosis. Anticancer Res. 2000;20(1A):407-16.

14. Meric F, Hunt K. Translation initiation in cancer: a novel target for therapy. Mol Cancer Ther. 2002;1(11):971-9. 